DR. GIACOMO EMMI (Orcid ID : 0000-0001-9575-8321)

Article type : Full Length

\title{
RITUXIMAB AS MAINTENANCE TREATMENT FOR SYSTEMIC LUPUS ERYTHEMATOSUS: A MULTICENTRE OBSERVATIONAL STUDY OF 147 PATIENTS
}

Matthias A. Cassia, $\mathrm{MD}^{1,2} *$; Federico Alberici, MD, $\mathrm{PhD}^{1,3}$; Rachel B. Jones, $\mathrm{MD}^{4}$; Rona $\mathrm{M}$. Smith, $\mathrm{MD}^{4}$; Giovanni Casazza, $\mathrm{PhD}^{2}$; Maria L. Urban, $\mathrm{MD}^{5}$; Giacomo Emmi, MD, $\mathrm{PhD}^{5}$; Gabriella Moroni, $\mathrm{MD}^{6}$; Renato A. Sinico, $\mathrm{MD}^{7}$; Piergiorgio Messa, $\mathrm{MD}^{6,8}$; Frances Hall, $\mathrm{MD}^{9}$; Augusto Vaglio, $\mathrm{MD}, \mathrm{PhD}^{10}$, Maurizio Gallieni, $\mathrm{MD}^{2,11}$; David R. Jayne, $\mathrm{MD}^{4}$

1: ASST Santi Paolo e Carlo, Nephrology Unit and Immunology Clinic, Milano, Italy

2: University of Milano, Dipartimento di Scienze Biomediche e Cliniche "L. Sacco", Milano, Italy

${ }^{3}$ : University of Milano, Dipartimento di Scienze della Salute, Milano, Italy

${ }^{4}$ : University of Cambridge, Department of Medicine, Cambridge, UK

${ }^{5}$ : University of Florence, Department of Experimental and Clinical Medicine, Florence, Italy

': Fondazione IRCCS Ca' Granda Ospedale Maggiore di Milano, Nephrology Unit, Milano, Italy

7. University of Milano Bicocca, Department of Medicine and Surgery, Milano, Italy

8: University of Milano, Dipartimento di Scienze Cliniche e di Comunità, Milano, Italy

${ }^{9}$ : Cambridge University Hospital, Rheumatology Unit, Cambridge, UK

${ }^{10}$ : University of Florence, Dipartimento di Scienze Biomediche, Sperimentali e Cliniche, Florence, Italy

${ }^{11}$ : ASST Fatebenefratelli Sacco

*Joint first Authors

Financial support and disclosures: The only financial support to disclose were travel grants from the patients' group “Associazione Malattie Autoimmuni Mario Rossi ONLUS".

MAC has received travel grants from the patients' group "Associazione Malattie Autoimmuni Mario Rossi ONLUS"; FA none; RBJ has received consultancy fees from Chemocentryx and research grants from GlaxoSmithKline; RMS lecture fees from Roche; GC none; MLU none; GE consultancy fees and advisory boards from Novartis, SOBI, GSK; GM none; RAS lecture fees from Roche; PM none; AV none; FH none; MG none; DJ has received research grants from Chemocentryx, GSK, Roche/Genentech and Genzyme/Sanofi, consulting fees from Astra-Zeneca, Boehringer-Ingelheim, Chemocentrx, InflaRx, Insmed and Takeda.

This article has been accepted for publication and undergone full peer review but has not been through the copyediting, typesetting, pagination and proofreading process, which may lead to differences between this version and the Version of Record. Please cite this article as doi: 10.1002/art.40932

This article is protected by copyright. All rights reserved. 


\section{Corresponding Author}

Federico Alberici MD, PhD

Assistant Professor - Consultant in Renal Medicine and Immunology Clinic

University of Milano - ASST Santi Paolo e Carlo, San Carlo Borromeo Hospital

20153 Milano, Italy

Tel. $+39(0) 240222489$

Fax. $+39(0) 240222222$

Mobile +393391203418

e-mail: federico.alberici@gmail.com

\section{Abstract}

Introduction: The efficacy of rituximab (RTX) in Systemic Lupus Erythematosus (SLE) is debated. We describe the outcomes of a European SLE cohort treated with RTX, with emphasis on its role as a maintenance agent.

Methods: All patients with SLE receiving RTX as induction across four centres were included, follow-up post RTX was collected including the subgroup treated with RTX as maintenance treatment (RMT). Disease flares during the follow-up were defined as an increase in disease activity and immunosuppressive drugs.

Results: Of 147 patients, at 6 months 27\% experienced treatment failure (TF); in a multivariate analysis, a low number of previous immunosuppressive therapies $(p=0.034)$ and low C4 levels $(p=0.008)$ reduced the risk of TF. Eighty patients received RMT over a median of 24.5 months during which 85 relapses, mainly musculoskeletal, were recorded (1.06 per patient); at the last RTX course, $84 \%$ of the patients were in remission. $28 / 80$ (35\%) patients never flared during the RMT with low damage accrual; active articular disease at the time of the first RTX course was associated with risk of flare during RMT ( $p=0.010)$. After RMT, relapse free survival was similar to patients receiving a single-RTX course $(p=0.72)$.

\section{Conclusions:}

RMT is a potential treatment option in difficult to treat patients. Relapses occur during RMT and are more likely in those with active articular disease at the time of the first RTX. Relapse risk after RMT remains high and apparently comparable to the one seen after a single-RTX course.

\section{INTRODUCTION}

Systemic Lupus Erythematosus (SLE) is an autoimmune disease characterised by clinical variability and complex pathogenesis $(1,2)$. B-cell depleting strategies have received continued attention in SLE(1) and, although rituximab (RTX) has proven effective in other autoimmune diseases characterised by hyperactivity of the B-cell compartment(3),

This article is protected by copyright. All rights reserved. 
randomised controlled trials (RCTs) exploring $\operatorname{RTX}$ in $\operatorname{SLE}(4,5)$ have failed to meet their primary end-points.

Sustained remission is the goal for disease management although infrequently achieved(6); improving the management of the maintenance phase of SLE is therefore an unmet need(7). Newer therapeutic options are needed and RTX is frequently employed in relapsing and refractory cases(8), its use is supported by retrospective and prospective non-randomised studies(1).

The discrepancy between the RCTs results and those observed in real-life settings suggests that solid predictors of response to RTX must be identified in order to better tailor the treatment strategies. Clinical predictors of response to RTX in SLE have been investigated, with heterogeneous results across different cohorts(9). Having severe disease, lack of haematological involvement or previous treatment with high dose steroids were associated with a good response in 116 patients(10); younger age and achievement of B-cell depletion six weeks after treatment with RTX were other favourable characteristics in a different population of 117 patients(11). Among biomarkers, the proportion of plasmablasts 6 months after RTX(11) and a single nucleotide polymorphism in the IL2/IL21 area have been associated with response(12); the former replicated in two different cohorts while the latter, although with a strong biological rationale(13), so far replicated only in a cohort of patients with microscopic polyangiitis(14).

This article is protected by copyright. All rights reserved. 
Importantly, in RTX treated patients time to relapse is highly variable(15, 16); relapses are associated to risk of damage accrual and therefore attention should be focused on flare prevention aiming at the right balance between immunosuppressive drug dosing, risk of toxicity and disease activity. RTX has a proven role as a maintenance agent in other autoimmune diseases(3) while in SLE, there is little published experience of repeat dose RTX(17-21). Of note the cohorts were small, the number of cycles low and the indications for repeating the treatment heterogeneous while no study has so far explored its use as maintenance treatment with the aim of relapse prevention.

In this study, we explore the efficacy and safety of RTX in a cohort of 147 patients with SLE with an emphasis on the subgroup of 80 patients re-treated with the drug as a maintenance agent in order to prevent relapses; prognostic factors associated with the response to RTX were also investigated.

\section{PATIENTS AND METHODS.}

Data on all patients with a diagnosis of SLE according to ACR or SLICC criteria that received at least one RTX administration from 2004 to 2016 were collected retrospectively at four centres: Addenbrooke's Hospital (Cambridge UK), ASST Santi Paolo e Carlo-San Carlo Borromeo Hospital (Milan IT), Policlinico Hospital (Milan IT) and Careggi Hospital (Florence IT). Ethical approval was obtained only at the Italian centres; it was not required in the UK centre due to the retrospective nature of the work.

This article is protected by copyright. All rights reserved. 


\section{Treatment protocol}

The patients were either treated with a "Single-RTX course" or with "RTX maintenance treatment" (RMT). "Single-RTX course" was any RTX dosing performed within a single month, such as $1 \mathrm{~g}$ or $375 \mathrm{mg} / \mathrm{m} 2$ given two weeks apart or for four consecutive weeks respectively. Patients receiving at least three "Single-RTX courses" with the aim of relapse prevention and with an interval of 4 to 8 months between consecutive treatments were classified as receiving RMT. Patients receiving RTX courses that did not meet this definition were censored at time of re-treatment. Treatment associated to RTX administration and data collected during the follow-up are reported in the supplementary methods. This a retrospective study, therefore no clear indications for treatment selection as well as RTX schedule within the RMT group was provided and it was result of the single physician experience and choice.

\section{Assessments}

Disease activity was assessed using the European Consensus Lupus Activity Measurement (ECLAM) score(22) since validated for retrospective studies(23), and the physician assessment (PA); the type and dose of immunosuppressive therapies was also recorded. PA was zero if no signs of active SLE were identified, 1 if the disease activity was mild and 2 if this was severe.

Response to the first RTX course assessed after six months was defined as complete response (CR), for PA of 0 or 1 , a reduction of ECLAM of at least $50 \%$ and decrease of the immune-modulating therapy (considering one between corticosteroids or immunesuppressors but no anti-malarials) by at least $25 \%$ from baseline; partial response (PR) for ECLAM reduction of $25-50 \%$, PA $>0$ and reduction of the immune-

This article is protected by copyright. All rights reserved. 
modulating therapy dose of $0-25 \%$. Any other case was defined as treatment failure $(\mathrm{TF})$.

Disease flares during RMT or after the first-last RTX course were defined as an increase in at least two of three parameters among PA, ECLAM, immunosuppressivecorticosteroids number/dose.

Patients were stratified in four groups according to disease severity: Mild, Moderate, Severe and Drug Sparing. The first three groups were defined according to the British Society of Rheumatology guidelines(8); the group "Drug Sparing" included patients with Mild or Moderate disease to whom RTX was administered with the aim of sparing an on-going immunosuppressive agent or avoiding an alternative immune suppressors contraindicated for the patient.

Late onset neutropenia (LON) definition and damage assessment methods are reported in the supplementary methods.

\section{Statistical Analysis}

Statistical analysis was performed using R (https://www.r-project.org), GraphPad Prism 7 (GraphPad Software, La Jolla, CA, USA) and SAS statistical software (version 9.4; SAS Institute, Cary, NC, USA). Results are expressed as count and percentage for categorical variables and median (interquartile range, IQR) or mean (95\% confidence interval, 95\% CI) for continuous variables.

This article is protected by copyright. All rights reserved. 
Methods for comparison of changes in variables and proportions of patients as well as for Univariate and multiple logistic regression are reported in the supplementary methods.

Time to relapse was assessed by the Kaplan-Meier survival analysis, and if comparisons between populations were required, the log-rank test was used. Two sided p-values $<0.05$, were considered statistically significant.

\section{RESULTS}

\section{Outcome after the first RTX}

The clinical characteristics at the time of the first RTX administration for the whole cohort are reported in Table 1. The mean (95\%CI) number of previous immunosuppressive drug employed in the four groups of disease severity Mild, Moderate, Severe and Drug Sparing was 2.92 (2.38-3.46), 2.74 (1.84-3.64), 2.38 (2.04$2.72)$ and $2.42(1.97-2.87)$ respectively.

Six months after the first RTX administration, 67 (45\%) patients were in CR and 41 in PR (28\%), while 39 (27\%) experienced TF. The ECLAM score declined from 4 at baseline (mean, 95\% CI 3.65-4.34) to 1.9 at 6 months $(1.66-2.14)(\mathrm{p}<0.0001)$; the prednisolone dose declined from a mean of 15.4 (95\%CI 13.1-17.7) to 8.45 (7.29-9.61) $(\mathrm{p}<0.0001)$. Within the four groups of disease severity Mild, Moderate, Severe and Drug Sparing the proportion of $\mathrm{TF}$ at 6 months was $65 \%, 50 \%, 25 \%$ and $6 \%$ respectively.

This article is protected by copyright. All rights reserved. 
At the multivariate analysis adjusted for confounding factors (other immunosuppressive drugs administered and prednisolone dose), the characteristics associated with the risk of experiencing TF were higher C4 levels (OR 1.76 (1.15-2.7), $\mathrm{p}=0.008)$ and the number of previous immunosuppressive agents (OR 7.77 (1.65-36.6), $\mathrm{p}=0.034)$ (Table S1).

\section{RTX Maintenance Treatment (RMT)}

80/147 (54\%) patients were treated with RMT; their characteristics are summarised in Table 1. Their median duration of maintenance treatment was 24 months (IQR 16-34; range 11-99 months), the median cumulative RTX dose was 6 gm (IQR 4.87-9 gm) and the median overall follow-up was 38 months (IQR 22-54). At the time of the first RTX administration, $69 / 80$ patients (86\%) were receiving a mean oral prednisolone dose of $11 \mathrm{mg} /$ day (95\%CI 9-13) and 46 (57\%) were receiving an immunosuppressive drug (not including hydroxychloroquine): 28 mycophenolate mofetil, 6 methotrexate, 4 azathioprine, 4 cyclophosphamide, 4 calcineurin inhibitors, 3 intravenous immunoglobulin and 4 other treatments. The mean ECLAM score at the first RTX was 3.5 (95\%CI 3.06-3.93) and the mean $(95 \% \mathrm{CI})$ number of previous immunosuppressive drug employed in the four groups of severity of disease Mild, Moderate, Severe and Drug Sparing were respectively 3.05 (2.28-3.82), 3.67 (2.7-4.64), 2.42 (2.06-2.78) and 2.14 (1.64-2.64). The different protocols employed for RTX administration within the RMT group are reported in the paragraph $\mathbf{R T X}$ schedule of administration within RMT of the supplementary material.

This article is protected by copyright. All rights reserved. 
After six months ECLAM declined to a mean of 1.86 (95\%CI 1.52-2.19) $(\mathrm{p}<0.0001)$ with a trend towards further reduction/stabilisation during maintenance treatment (figure 1); at the six month assessment $30 / 80$ patients (38\%) were in CR and this proportion increased to $40-51$ and $48 \%$ respectively at the 12,18 and 24 month assessment. The proportion of patients not taking corticosteroids increased from $14 \%$ to $41 \%$ while the mean dose of prednisolone dropped from $11 \mathrm{mg} /$ day (95\%CI 8.9-13) at RTX initiation to $3.8(95 \%$ CI $1.1-6.4)$ by the $6^{\text {th }}$ administration; a trend towards a reduction of the proportion of patients receiving other immunosuppressive drugs was also observed (figure 1).

$52 / 80(65 \%)$ patients experienced 85 relapses $(1.06$ relapses per patient, 53 per 100patient year) after a median of 11.5 months (IQR 8-18.25); relapses were more frequent between 6-12 months after the first RTX administration (Table S2) and more frequently involved joints (66\%) and skin (40\%) (Table S3). At the time of flare, the mean ECLAM score was 2.72 (95\%CI 2.42-3.03) and its mean increase compared to the previous assessment was 1.42 (95\%CI 1.11-1.74). Relapses were managed with an increase in prednisolone dose in $29 / 85$ cases (34\%) [mean increase $11.1 \mathrm{mg}$ (95\%CI 6.9-15.4)], with a new immunosuppressive drug in 22/85 (26\%), with an increase in the dose of an on-going immunosuppressive drug in 12/85 (14\%), 7/85 (8\%) patients were managed by bringing forward the pre-planned RTX administration while in 15/85 (18\%) the flare was managed by waiting for the pre-planned RTX infusion.

At the time of the last RTX administration, $67 / 80$ (84\%) of the patients were in remission, the mean ECLAM score was 1.5 (IQR 1.13-1.86) and the mean prednisolone dose was $6.35 \mathrm{mg} /$ day (95\%CI 4.8-7.9).

This article is protected by copyright. All rights reserved. 


\section{Outcome after the last RTX infusion}

Of the 67 patients that received a single-RTX course, $54(80 \%)$ experienced a CR or PR at the sixth month assessment and were therefore followed for post therapy flare; the median follow-up after the sixth month assessment was 7.3 months (IQR 3.46-9.78). Eighteen flares (33\%) were observed (51.5 flares per 100-patient year); the mean ECLAM score for the flaring patients was 2.94 (95\%CI 1.97-3.91); the sites showing active disease and the flare management are shown in Table $\mathbf{S 4 .}$

Of the 80 patients receiving RMT, post-treatment follow-up was available in 57 (71\%); the median follow-up after the last RTX was 13 months (IQR 4-22). Thirty (53\%) flares were observed (38 flares per 100-patient year); the mean ECLAM score for flaring patients was 3.09 (95\% CI 2.6-3.58); the sites showing active disease and flare management are shown in Table S4.

The rate of flares involving major organs during RMT was 38\%, this increased to $67 \%$ when RMT was withdrawn ( $\mathrm{p}=0.01)$; in particular, the flare rate per 100 patient-years involving neurological, pulmonary and haematological systems after RMT increased compared to the one observed during RMT while the renal ones remained stable (figure 2). Of note the number of flares adjusted per number of patients at risk remained roughly stable during the follow-up (Table S5).

The median relapse-free survival after the last RTX infusion was 16 months in the cohort treated with a single-RTX course (IQR 1.2-9) and 17 months in the RMT cohort (IQR 4-22) ( $\mathrm{p=0.72);} \mathrm{a} \mathrm{trend} \mathrm{towards} \mathrm{a} \mathrm{longer} \mathrm{relapse-free} \mathrm{survival} \mathrm{was} \mathrm{noted} \mathrm{when}$ considering only non-musculoskeletal flares although the difference was non significant $(\mathrm{p}=0.10)$ (figure 3$)$.

This article is protected by copyright. All rights reserved. 


\section{Sustained responders}

Among the 80 patients of the RMT cohort, 28 (35\%) did not experience any flare during the maintenance period ("Sustained responders"). When comparing the group of the "Sustained responders" to those with at least one flare, the presence of active articular disease at the time of the first RTX administration was at multivariate analysis associated with the risk of flare [OR 3.55 (95\%CI 1.34-9.37) p=0.010] (Table 2) even after adjusting the analyses for the disease severity considered as a potential a priori confounder [OR 3.38 (95\%CI 1.25-9.11) p=0.016].

Post-RMT follow-up was available for $21 / 28$ of the Sustained responders (75\%); the median follow-up was 17.5 months (IQR 5.3-23.8). 10/21 patients (48\%) flared (27 flares per 100-patient year); the mean ECLAM score for flaring patients was 2.45 (95\%CI 1.64-3.27); sites showing active disease at the time of the flare were joints in 5 (50\%), haematological in 4 (40\%), renal and CNS in 2 (20\%) and skin in $1(10 \%)$. Flares were managed by increasing the prednisolone dose in $4(40 \%)$, starting a new immunosuppressive drug in $4(40 \%)$, increasing the dose of an on-going immunosuppressive drug in $1(10 \%)$ and in three patients (30\%) no treatment changes were recorded.

\section{Severe Adverse events}

One hundred and nine severe adverse events (SAEs) occurred in 54 patients during the follow-up, with a rate of 30 SAEs per 100-patient year; in the RMT subgroup the rate was 24 while in the single-RTX subgroup it was 53 (Table S6); the incidence of malignant neoplasms was $4 \%$ in the overall population and 3\% in the RMT group. The

This article is protected by copyright. All rights reserved. 
cumulative number and the proportions of the different SAEs did not differ between the patients treated with RMT and those treated with a single-RTX course (data not shown).

Sixteen episodes of LON were recorded during the 452 RTX administrations (4\%) in 9/80 patients (11\%) with four patients experiencing two or more episodes; ten episodes were of grade II, 4 of grade III and 2 of grade IV. In two patients the first episode of LON happened after the first RTX administration while in seven after one of the following cycles; the median time from the last RTX administration and the LON detection has been of 8 months (IQR 5-11). Of the two patients experiencing grade IV LON, one died of neutropenic sepsis and one was admitted for clinical monitoring without experiencing any infection or requiring any specific therapy.

Eleven patients died during follow-up, 2/67 (3\%) in the group that did not receive RMT and nine $(9 / 80,11.3 \%)$ in the RMT group; in the latter group seven died during RMT and two after (respectively 19 and 35 months after the last RTX infusion). Details regarding the cause of death are reported in Table $\boldsymbol{S 7 .}$

\section{Damage}

At the time of the first RTX administration, the mean SLICC score was 1.69 (95\%CI 1.44$1.94)$ in the overall population and $1.8(95 \% \mathrm{CI} 1.46-2.14)$ in the RMT group. Damage increased during the follow-up with a mean SLICC score at the last available follow-up of 2.14 (95\%CI 1.83-2.44).

This article is protected by copyright. All rights reserved. 
The increase in SLICC per patient per year pre-RTX administration was 0.21 while during the RMT it was 0.23; in the "Sustained responders" subgroup the increase in the SLICC per patient per year was 0.17 .

\section{Laboratory parameters}

During RMT C3 and C4 levels remained stable if they were normal at the time of the first RTX administration (figure 4); in the subgroup of patients with reduced levels the values increased to normal and then stabilised (figure S1). IgG levels remained stable during the RMT whereas a trend towards reduction for IgM was noted (figure 4). AntiDNA levels assessed with ELISA decreased after the first RTX to fall within the normal range and to remain stable during the RMT (figure S2). CD19+ B-cells were undetectable in $76 \%$ of the patients at the time of the second RTX and tended to remain the same during follow-up (figure S3).

\section{DISCUSSION}

SLE remains a challenging disease being characterised by a chronic relapsing course despite the availability of multiple therapies. Although there have been no prospective trials showing superiority of RTX over placebo $(4,5)$, this drug is widely used. Our cohort of 147 patients treated with RTX is one of the largest published so far(24) and the overall positive response further supports the role of this drug in SLE management.

Patients with low C4, with a lower number of previous immunosuppressive drugs and with severe disease were more likely to respond favourably: this would suggest that the ideal candidates for RTX may be those with more active disease and without a clear refractory course. This is consistent with results from other studies $(10,11)$.

This article is protected by copyright. All rights reserved. 
Despite the challenges deriving from the management of active SLE, the maintenance phase plays a critical role in the long-term outcome, influencing damage accrual(7). In RTX treated patients time to relapse is highly variable $(15,16)$ and a RTX based maintenance treatment at fixed intervals may be attractive.

Our study shows for the first time that RMT may be an option in SLE. According to our data, disease activity remains stable over time while allowing reduction of immunosuppressive drug; overall corticosteroids dose reduced after the first RTX cycle and the proportion of patients able to withdraw immunesoppressors at the end of the RMT was 41\%. However, disease reactivation was common; flares were generally not severe, being mainly musculoskeletal and requiring only minor therapeutic changes. Importantly during RMT severe flares were rare while these increased when RMT was suspended: in particular neurological, pulmonary and haematological reactivation doubled.

Interestingly, despite improved disease control with RMT and reduction in corticosteroid and immunosuppressive drug dosing, damage accumulated at the same rate as before the RMT phase. Damage in SLE is more likely to increase in patients having already experienced it as well as in the ones with renal involvement(25); moreover corticosteroids may cause late onset damage(26). Of interest, in an every-day clinical life setting study of 188 patients treated with belimumab, damage accrual rate decreased during the therapy although in the context of a less severe disease and lower damage at the beginning of the therapy (mean SLICC score of $0.85+/-1.11$ compared to $1.8+/-0.34$ of this RMT cohort)(27). The RMT cohort consisted of patients with severe disease in $50 \%$ and active renal disease in $20 \%$, with a long disease course and a 
significant damage burden already recorded at time of the first RTX; in this context further damage progression may not be surprising. It will be of interest to observe in other cohorts with lower damage if this may be confirmed as well as exploring belimumab role on damage prevention even in groups with a high SLICC score.

Among all patients treated with RMT, 35\% were classified as "Sustained responders" as they did not experience any disease reactivation during the maintenance course; this group was characterised by lower damage accumulation compared to the rest of the population and less frequent articular involvement at the time of the first RTX administration. However, apart disease severity, no other confounders has been considered in the univariate and multivariate analyses and this result should therefore be interpreted with caution. No other factors associated to the risk of flares during RMT have been identified and future studies will need to focus on this particular aspect.

After RMT the relapse free survival remains the same compared to that for patients treated with a single-RTX course even when excluding musculoskeletal flares. If confirmed, this result would not be consistent with the experience of RMT in AAV(28). This data, although of interest, should be interpret with caution being the result of a comparison performed between two heterogeneous and non-matched groups; moreover, the RMT cohort was probably represented by patients with a more severe disease profile as suggested by the higher proportion of TF 6 months after the first RTX administration compared to the Single-RTX group. Importantly, relapse rate after RMT, may have been influenced by the natural course of the disease since no adjustment for this has been performed in our analyses; the role of RMT suspension on post RMT flares

This article is protected by copyright. All rights reserved. 
has therefore to be confirmed. However reassuringly a clear trend for increase of the relapse rate during the follow-up has not been identified.

The mortality observed in our cohort is within the high range of what described in literature especially in the RMT group (11.3\%): we feel that this may be consequence of the high risk profile of this population. Of note, patients with severe comorbidities are usually excluded from RCTs and the mortality rate in studies including patients with similar profile of severity is around 8\%(29). Reassuringly, cancer risk was similar to the one described in the literature.

Importantly, LON incidence in the RMT group (4\% of the RTX administrations in $11 \%$ of the patients) was higher than what described in other cohorts of RTX treated patients with heterogeneous immunological diseases $(2 \%)(30)$ but lower than what described in other SLE cohorts (29.9\%)(31). Only two episodes observed in the RMT group happened after the first RTX administration while the remaining 14 were recorded after subsequent administrations suggesting a possible role for repeat RTX in increasing LON risk; four patients experienced two or more episodes. Of note, one patient of the RMT cohort died of neutropenic sepsis.

Our study is a multicentre - multidisciplinary survey involving a large cohort collected from tertiary care centres with experience in managing SLE. However, when interpreting these results, several observations have to be made. As a retrospective study, some heterogeneity across different centres must be taken into account as shown by the variability of the treatment schemes during RMT; moreover only $89 \%$ of the population followed a pre-planned RTX scheme of administration while $9 \%$ entered a 
fixed interval scheme only within 8 months from the first RTX administration and 2\% were treated irregularly according to changes in symptoms and serology; importantly $11 \%$ of the patients supposed to be treated regularly missed at least one administration during the first 24 months of treatment. All these factors, in the context of a highly variable timing of relapse in RTX-treated SLE patients $(15,16)$, may have contributed to the high flare rate observed in our population. Whether this RMT protocol should be considered as a genuine relapse prevention approach rather than a repeat induction is unclear and this will need to be tested in a prospective fashion. Importantly, posttreatment follow-up was relatively short and conclusions regarding this phase of the study should be interpreted with caution.

Of note, $51 / 147$ (35\%) patients had articular and mucocuteneous manifestations as prominent feature of active SLE at the moment of the first RTX; all of these patients had received corticosteroids and hydroxycloroquine in their past as well as at least one drug among MMF, MTX and AZA with 39\% having received two of them. Despite the proportion of patients treated with MTX in our cohort was higher compared to the BLISS trials $(7-21.2 \%)(32,33)$, still only $50 \%$ and $41 \%$ of the potentially eligible patients with respectively articular and mucocutanous disease received this drug in their history. This may reflect a more limited experience with this drug of the nephrologists involved in the study as well as safety concerns related to MTX use(34). Moreover, according to the timing of reimbursement approval from the Italian and UK Health Systems, 5\% of our cohort would have been eligible for belimumab and was however treated with RTX; this may have been consequence of a greater experience with RTX of the investigators involved in this study. However, reassuringly, no centereffect was noted for RTX, MTX and belimumab prescription. The high rate of patients 
with musculoskeletal and skin involvement may be the reason of the relatively high proportion of patients with mild disease treated with RTX in our population (26\%); this group, according to the guidelines of the British Society of Rheumatology(8), should not be treated with RTX. However it is to be noted that for patients with less severe disease, RTX was employed in the context of reduced therapeutic options having this population previously failed a mean of around 3 immunosuppressive drugs. Another limitation is that for $18 \%$ of the patients, a single-RTX cycle had been employed before the referral to the centers of this study: at the moment of the inclusion in our survey however all these patients were B-cell repopulated. Our population might therefore not necessarily reflect other centers or nowadays clinical practice especially with belimumab being more easily available.

The response criteria employed are original and not validated; however response definitions in retrospective RTX treated SLE cohorts have been historically heterogeneous and reassuringly in our population response rates are in keeping with the literature(1). Together with the change of a semi-objective score such as ECLAM, PA has been employed due to the central role of the physician judgment for the assessment of a complex disease such as SLE while the immunesuppressant dose reduction has been included since in this cohort RTX has been frequently employed with the aim of sparing other drugs.

In conclusion, we confirm in a large multicentre cohort that RTX may be an option in patients with SLE. Its use as maintenance treatment allows corticosteroid and immunosuppressive drug sparing while stabilising the overall disease activity; however is to be noted that musculoskeletal flares were common. Patients without articular 
involvement at the time of the first RTX were less likely to experience relapses during RMT. At the time of drug withdrawal, relapse free survival remains apparently comparable to the one of patients treated with a single-RTX course. A RMT regimen might be considered for patients with severe disease-related complications, for whom waiting for the relapse in order to treat on demand may be risky, and when first-line immunosuppressive options are exhausted.

\section{Acknowledgments}

We gratefully acknowledge Prof. Massimiliano Romanelli, Prof. Manuela Nebuloni, Prof. Giovanna Mantovani and Prof. Nicola Montano (council members of the MD/PhD program of the University of Milan) and the patients' group "Associazione Malattie Autoimmuni Mario Rossi ONLUS" for the support to MC during the development of the research project.

\section{References}

1. Cassia M, Alberici F, Gallieni M, Jayne D. Lupus nephritis and B-cell targeting therapy. Expert Rev Clin Immunol. 2017;13(10):951-62.

2. Tsokos GC, Lo MS, Costa Reis P, Sullivan KE. New insights into the immunopathogenesis of systemic lupus erythematosus. Nat Rev Rheumatol. 2016;12(12):716-30.

3. Guillevin L, Pagnoux C, Karras A, Khouatra C, Aumaitre O, Cohen P, et al. Rituximab versus azathioprine for maintenance in ANCA-associated vasculitis. N Engl J Med. 2014;371(19):1771-80.

4. Rovin BH, Furie R, Latinis K, Looney RJ, Fervenza FC, Sanchez-Guerrero J, et al. Efficacy and safety of rituximab in patients with active proliferative lupus nephritis: the Lupus Nephritis Assessment with Rituximab study. Arthritis Rheum. 2012;64(4):121526.

This article is protected by copyright. All rights reserved. 
5. Merrill JT, Neuwelt CM, Wallace DJ, Shanahan JC, Latinis KM, Oates JC, et al. Efficacy and safety of rituximab in moderately-to-severely active systemic lupus erythematosus: the randomized, double-blind, phase II/III systemic lupus erythematosus evaluation of rituximab trial. Arthritis Rheum. 2010;62(1):222-33.

6. Kim M, Merrill J, Kalunian K, Hahn B, Roach A, Izmirly P, et al. Brief Report: Longitudinal Patterns of Response to Standard of Care Therapy for Systemic Lupus Erythematosus: Implications for Clinical Trial Design. Arthritis Rheumatol. 2017;69(4):785-90.

7. Watson P, Brennan A, Birch H, Fang H, Petri M. An integrated extrapolation of long-term outcomes in systemic lupus erythematosus: analysis and simulation of the Hopkins lupus cohort. Rheumatology (Oxford). 2015;54(4):623-32.

8. Gordon C, Amissah-Arthur MB, Gayed M, Brown S, Bruce IN, D'Cruz D, et al. The British Society for Rheumatology guideline for the management of systemic lupus erythematosus in adults. Rheumatology (Oxford). 2018;57(1):e1-e45.

9. Pirone C, Mendoza-Pinto C, van der Windt DA, Parker B, M OS, Bruce IN. Predictive and prognostic factors influencing outcomes of rituximab therapy in systemic lupus erythematosus (SLE): A systematic review. Semin Arthritis Rheum. $2017 ; 47(3): 384-96$.

10. Fernandez-Nebro A, de la Fuente JL, Carreno L, Izquierdo MG, Tomero E, RuaFigueroa I, et al. Multicenter longitudinal study of B-lymphocyte depletion in refractory systemic lupus erythematosus: the LESIMAB study. Lupus. 2012;21(10):1063-76.

11. Md Yusof MY, Shaw D, El-Sherbiny YM, Dunn E, Rawstron AC, Emery P, et al. Predicting and managing primary and secondary non-response to rituximab using B-cell biomarkers in systemic lupus erythematosus. Ann Rheum Dis. 2017;76(11):1829-36.

This article is protected by copyright. All rights reserved. 
12. Marquez A, Davila-Fajardo CL, Robledo G, Rubio JL, de Ramon Garrido E, GarciaHernandez FJ, et al. IL2/IL21 region polymorphism influences response to rituximab in systemic lupus erythematosus patients. Mol Biol Rep. 2013;40(8):4851-6.

13. Wang S, Wang J, Kumar V, Karnell JL, Naiman B, Gross PS, et al. IL-21 drives expansion and plasma cell differentiation of autoreactive CD11c(hi)T-bet(+) B cells in SLE. Nat Commun. 2018;9(1):1758.

14. Alberici F, Smith RM, Fonseca M, Willcocks LC, Jones RB, Holle JU, et al. Association of a TNFSF13B (BAFF) regulatory region single nucleotide polymorphism with response to rituximab in antineutrophil cytoplasmic antibody-associated vasculitis. J Allergy Clin Immunol. 2017;139(5):1684-7 e10.

15. Vital EM, Dass S, Buch MH, Henshaw K, Pease CT, Martin MF, et al. B cell biomarkers of rituximab responses in systemic lupus erythematosus. Arthritis Rheum. 2011;63(10):3038-47.

16. Ng KP, Cambridge G, Leandro MJ, Edwards JC, Ehrenstein M, Isenberg DA. B cell depletion therapy in systemic lupus erythematosus: long-term follow-up and predictors of response. Ann Rheum Dis. 2007;66(9):1259-62.

17. Weide R, Heymanns J, Pandorf A, Koppler H. Successful long-term treatment of systemic lupus erythematosus with rituximab maintenance therapy. Lupus. 2003;12(10):779-82.

18. Turner-Stokes T, Lu TY, Ehrenstein MR, Giles I, Rahman A, Isenberg DA. The efficacy of repeated treatment with B-cell depletion therapy in systemic lupus erythematosus: an evaluation. Rheumatology (Oxford). 2011;50(8):1401-8.

19. Aguiar R, Araujo C, Martins-Coelho G, Isenberg D. Use of Rituximab in Systemic Lupus Erythematosus: A Single Center Experience Over 14 Years. Arthritis Care Res (Hoboken). 2017;69(2):257-62.

This article is protected by copyright. All rights reserved. 
20. Terrier B, Amoura Z, Ravaud P, Hachulla E, Jouenne R, Combe B, et al. Safety and efficacy of rituximab in systemic lupus erythematosus: results from 136 patients from the French AutoImmunity and Rituximab registry. Arthritis Rheum. 2010;62(8):245866.

21. Emmi G, Urban ML, Scalera A, Becatti M, Fiorillo C, Silvestri E, et al. Repeated low-dose courses of rituximab in SLE-associated antiphospholipid syndrome: Data from a tertiary dedicated centre. Semin Arthritis Rheum. 2017;46(4):e21-e3.

22. Vitali C, Bencivelli W, Isenberg DA, Smolen JS, Snaith ML, Sciuto M, et al. Disease activity in systemic lupus erythematosus: report of the Consensus Study Group of the European Workshop for Rheumatology Research. II. Identification of the variables indicative of disease activity and their use in the development of an activity score. The European Consensus Study Group for Disease Activity in SLE. Clin Exp Rheumatol. 1992;10(5):541-7.

23. Mosca M, Bencivelli W, Vitali C, Carrai P, Neri R, Bombardieri S. The validity of the ECLAM index for the retrospective evaluation of disease activity in systemic lupus erythematosus. Lupus. 2000;9(6):445-50.

24. McCarthy EM, Sutton E, Nesbit S, White J, Parker B, Jayne D, et al. Short-term efficacy and safety of rituximab therapy in refractory systemic lupus erythematosus: results from the British Isles Lupus Assessment Group Biologics Register. Rheumatology (Oxford). 2018;57(3):470-9.

25. Bruce IN, O'Keeffe AG, Farewell V, Hanly JG, Manzi S, Su L, et al. Factors associated with damage accrual in patients with systemic lupus erythematosus: results from the Systemic Lupus International Collaborating Clinics (SLICC) Inception Cohort. Ann Rheum Dis. 2015;74(9):1706-13.

This article is protected by copyright. All rights reserved. 
26. Gladman DD, Urowitz MB, Rahman P, Ibanez D, Tam LS. Accrual of organ damage over time in patients with systemic lupus erythematosus. J Rheumatol. 2003;30(9):1955-9.

27. Iaccarino L, Andreoli L, Bocci EB, Bortoluzzi A, Ceccarelli F, Conti F, et al. Clinical predictors of response and discontinuation of belimumab in patients with systemic lupus erythematosus in real life setting. Results of a large, multicentric, nationwide study. J Autoimmun. 2018;86:1-8.

28. Alberici F, Smith RM, Jones RB, Roberts DM, Willcocks LC, Chaudhry A, et al. Long-term follow-up of patients who received repeat-dose rituximab as maintenance therapy for ANCA-associated vasculitis. Rheumatology (Oxford). 2015;54(7):1153-60.

29. Doria A, Iaccarino L, Ghirardello A, Zampieri S, Arienti S, Sarzi-Puttini P, et al. Long-term prognosis and causes of death in systemic lupus erythematosus. Am J Med. 2006;119(8):700-6.

30. Salmon JH, Cacoub P, Combe B, Sibilia J, Pallot-Prades B, Fain O, et al. Late-onset neutropenia after treatment with rituximab for rheumatoid arthritis and other autoimmune diseases: data from the AutoImmunity and Rituximab registry. RMD Open. 2015;1(1):e000034.

31. Parodis I, Soder F, Faustini F, Kasza Z, Samuelsson I, Zickert A, et al. Rituximabmediated late-onset neutropenia in systemic lupus erythematosus - distinct roles of BAFF and APRIL. Lupus. 2018;27(9):1470-8.

32. Navarra SV, Guzman RM, Gallacher AE, Hall S, Levy RA, Jimenez RE, et al. Efficacy and safety of belimumab in patients with active systemic lupus erythematosus: a randomised, placebo-controlled, phase 3 trial. Lancet. 2011;377(9767):721-31.

33. Furie R, Petri M, Zamani O, Cervera R, Wallace DJ, Tegzova D, et al. A phase III, randomized, placebo-controlled study of belimumab, a monoclonal antibody that 
inhibits B lymphocyte stimulator, in patients with systemic lupus erythematosus. Arthritis Rheum. 2011;63(12):3918-30.

34. Vial T, Patat AM, Boels D, Castellan D, Villa A, Theophile H, et al. Adverse consequences of low-dose methotrexate medication errors: data from French poison control and pharmacovigilance centers. Joint Bone Spine. 2018.

This article is protected by copyright. All rights reserved. 
TABLES AND FIGURES

Table 1. Main characteristics of the 147 SLE patients treated with RTX..

\begin{tabular}{|c|c|c|c|}
\hline & RMT $(n=80)$ & $\begin{array}{l}\text { Single-RTX } \\
(\mathrm{n}=67)\end{array}$ & $\begin{array}{l}\text { Overall cohort } \\
(n=147)\end{array}$ \\
\hline Age (years) & $45(42-48)$ & $42(39-46)$ & $44(41-46)$ \\
\hline Gender - Female & $71(90 \%)$ & $63(94 \%)$ & $134(91 \%)$ \\
\hline Ethnicity - Caucasian & $74(93 \%)$ & $62(93 \%)$ & $136(93 \%)$ \\
\hline Prior disease duration (months) & $154(100-208)$ & $114(87-141)$ & $133(104-161)$ \\
\hline RTX given at the time of diagnoses & $3(4 \%)$ & $4(6 \%)$ & $7(5 \%)$ \\
\hline \multicolumn{4}{|l|}{$\begin{array}{l}\text { Organ manifestations at first RTX } \\
\text { administration }\end{array}$} \\
\hline Musculoskeletal & $52(65 \%)$ & $35(53 \%)$ & $87(60 \%)$ \\
\hline Skin & $49(61 \%)$ & $31(46 \%)$ & $80(54 \%)$ \\
\hline Haematological & $26(33 \%)$ & $37(55 \%)$ & $63(43 \%)$ \\
\hline Pulmonary & $18(23 \%)$ & $6(9 \%)$ & $24(16 \%)$ \\
\hline Renal & $15(19 \%)$ & $37(55 \%)$ & $52(36 \%)$ \\
\hline Neurological & $14(18 \%)$ & $8(12 \%)$ & $22(15 \%)$ \\
\hline \multicolumn{4}{|l|}{ Previous therapies } \\
\hline Mycophenolate mofetil & $67(84 \%)$ & $42(63 \%)$ & $109(74 \%)$ \\
\hline Hydroxychloroquine & $61(76 \%)$ & $38(57 \%)$ & $99(67 \%)$ \\
\hline Azathioprine & $41(51 \%)$ & $26(39 \%)$ & $67(46 \%)$ \\
\hline Methotrexate & $26(33 \%)$ & $13(19 \%)$ & $39(27 \%)$ \\
\hline Cyclophosphamide & $24(30 \%)$ & $21(31 \%)$ & $45(31 \%)$ \\
\hline Rituximab & $14(18 \%)$ & $12(18 \%)$ & $26(18 \%)$ \\
\hline Calcineurin inhibitor & $11(14 \%)$ & $24(36 \%)$ & $35(24 \%)$ \\
\hline Anti-TNF therapy & $6(8 \%)$ & $3(4 \%)$ & $9(6 \%)$ \\
\hline Plasma exchange & $4(5 \%)$ & $2(3 \%)$ & $6(4 \%)$ \\
\hline IV immunoglobulins & $4(5 \%)$ & $6(9 \%)$ & $10(7 \%)$ \\
\hline Other & $10(13 \%)$ & $9(13 \%)$ & $19(13 \%)$ \\
\hline $\begin{array}{l}\text { Previous immunosuppressive } \\
\text { therapies per patient } €\end{array}$ & $4.3(4-4.7)$ & $3.9(3.5-4.4)$ & $4.1(3.9-4.4)$ \\
\hline \multicolumn{4}{|l|}{ Severity of Disease } \\
\hline Mild & $20(25 \%)$ & $4(6 \%)$ & $24(16 \%)$ \\
\hline Moderate & $6(7 \%)$ & $17(25 \%)$ & $23(16 \%)$ \\
\hline Severe & $40(50 \%)$ & $34(51 \%)$ & $74(50 \%)$ \\
\hline Drug Sparing & $14(18 \%)$ & $12(18 \%)$ & $26(18 \%)$ \\
\hline \multicolumn{4}{|l|}{ Laboratory Data } \\
\hline Anti-DNA level (IU/mL) & $67.2(31.9-104.5)$ & $75.4(39-111.5)$ & $71(46-96)$ \\
\hline C3 (g/L) & $1.1(1.02-1.21)$ & $0.85(0.75-94)$ & $1(0.92-1.07)$ \\
\hline $\mathrm{C} 4(\mathrm{~g} / \mathrm{L})$ & $0.2(0.18-0.24)$ & $0.15(0.12-0.18)$ & $0.18(0.16-0.2)$ \\
\hline \multicolumn{4}{|l|}{ Response to RTX at 6 months } \\
\hline Complete Response & $32(40 \%)$ & $35(52 \%)$ & $67(45 \%)$ \\
\hline Partial Response & $21(26 \%)$ & $20(30 \%)$ & $41(28 \%)$ \\
\hline Treatment Failure & $27(34 \%)$ & $12(18 \%)$ & $39(27 \%)$ \\
\hline
\end{tabular}

RMT: Rituximab Maintenance Treatment

Results are expressed as $\mathrm{n} / \mathrm{N}(\%)$ for categorical variables and mean (95\%CI) for continuous variables.

$£$ Includes also hydroxychloroquine

This article is protected by copyright. All rights reserved. 
Table 2. Clinical characteristics and their association with the risk of experiencing at least one flare during the RMT at univariate and multivariate analyses

\begin{tabular}{|c|c|c|c|c|}
\hline & \multicolumn{2}{|l|}{ Univariate } & \multicolumn{2}{|c|}{ Multivariate } \\
\hline Variable & Odds ratio & $\mathbf{p}$ & Odds ratio & $\mathbf{p}$ \\
\hline Gender & & 0.150 & - & - \\
\hline Female (reference) & 1 & & & \\
\hline Male & $4.8(0.57-40.5)$ & & & \\
\hline $\begin{array}{l}\text { Number of immunosuppressive drugs } \\
\text { before RTX }\end{array}$ & & 0.650 & - & - \\
\hline $0-2$ (reference) & 1 & & & \\
\hline $3-4$ & $1.51(0.51-4.46)$ & & & \\
\hline$>4$ & $0.93(0.22-4)$ & & & \\
\hline Physician Assessment at RTX & & 0.501 & - & - \\
\hline 0 & 1 & & & \\
\hline 1 & $2.81(0.39-20.4)$ & & & \\
\hline 2 & $3.08(0.47-20.23)$ & & & \\
\hline \multicolumn{5}{|l|}{ Clinical manifestations at RTX } \\
\hline Systemic & $2.00(0.77-5.16)$ & 0.150 & - & - \\
\hline Articular & 3.55 (1.34-9.37) & 0.011 & 3.55 (1.34-9.37) & 0.011 \\
\hline Skin & $2.67(1.04-6.87)$ & 0.042 & - & - \\
\hline Muscle & $0.51(0.07-3.83)$ & 0.512 & - & - \\
\hline Pulmonary & $1.65(0.53-5.18)$ & 0.390 & - & - \\
\hline CNS & $2.18(0.55-8.58)$ & 0.264 & - & - \\
\hline Renal & $0.56(0.18-1.754)$ & 0.316 & - & - \\
\hline Hematologic & $0.64(0.24-1.70)$ & 0.373 & - & - \\
\hline ECLAM score at RTX $\$$ & $1.02(0.80-1.29)$ & 0.887 & - & - \\
\hline \multicolumn{5}{|l|}{ Laboratory Values at RTX } \\
\hline Anti-DNA titre $^{\$}$ & $1.001(0.997-1.006)$ & 0.615 & - & - \\
\hline $\mathrm{C}^{\circ}$ & $1.02(0.89-1.17)$ & 0.786 & - & - \\
\hline $\mathrm{C} 4^{\circ}$ & $1.21(0.72-2.05)$ & 0.474 & - & - \\
\hline Other immunosuppressors with RTX & $1(0.47-2.09)$ & 0.993 & - & - \\
\hline Dose of prednisolone at the time of RTX & $0.95(0.91-1.01)$ & 0.079 & - & - \\
\hline
\end{tabular}

${ }^{\circ}$ Odds ratio expressed for 0.1 increase

$\$$ Odds ratio expressed for unit increase of the independent variable

This article is protected by copyright. All rights reserved. 
Figure 1. Changes of the ECLAM score, prednisolone and immunosuppressive treatment in the 80 SLE patients who received RTX Maintenance Treatment (RMT) for relapse prevention.

Panel A. Data are shown as mean with whiskers representing the $95 \%$ confidence interval. The grey area includes the part of the follow-up after the first RTX administration. The * indicates a reduction of ECLAM score statistically significant compared to baseline

Panel B. Bars show the proportion of patients either receiving (light grey) or not receiving (dark grey) other immunosuppressive drugs at the moment of the first RTX administration and during the RMT; the line represents the changes in the mean $(95 \% \mathrm{CI})$ prednisolone (PDN) dose. The star highlights a significant difference compared to baseline $(\mathrm{p}<0.05)$.

Figure 2. Major organ involvement at the time of flare during and after RTX Maintenance Treatment (RMT)

Figure 3. Relapse free survival of two cohorts of patients with SLE after the last RTX administration.

The red curve is showing the relapse free survival in a group of patients treated with a single-RTX course, the green curve the relapse free survival in cohort treated with RTX Maintenance Treatment (RMT) after the last RTX course.

Panel A refers to the overall population, Panel B included as event only the nonmusculoskeletal flares.

Figure 4. Complement and immunoglobulin levels during follow-up in a cohort of 80 SLE patients receiving RTX Maintenance Treatment (RMT) with the aim of relapse prevention.

Data are provided as mean, error bars represent 95\%CI. The grey area of the figure represents values within the normal range. The star highlights a significant difference compared to baseline $(\mathrm{p}<0.05)$.

This article is protected by copyright. All rights reserved. 
A

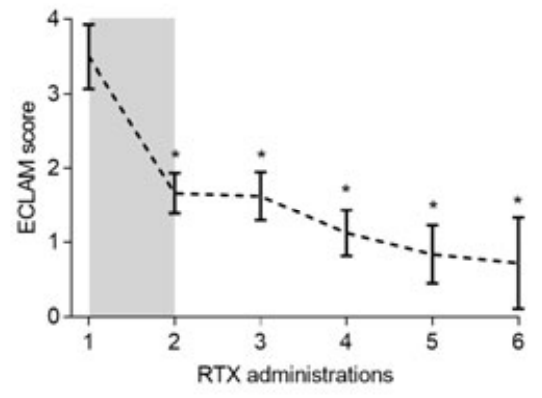

$\begin{array}{lllllll}\text { Number of patients } & 80 & 80 & 80 & 57 & 42 & 30\end{array}$

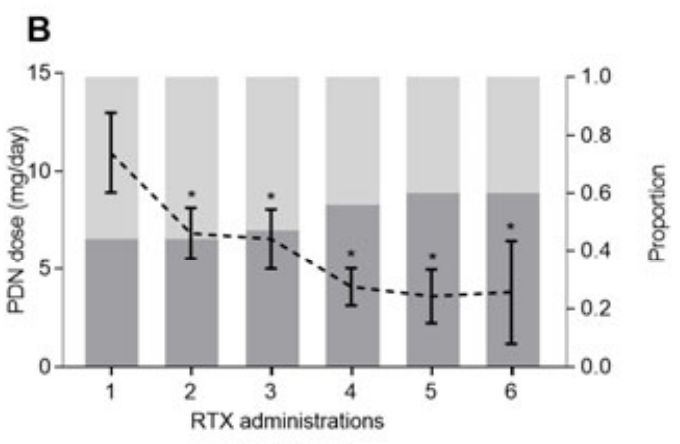

$\begin{array}{llllll}80 & 80 & 80 & 57 & 42 & 30\end{array}$



This article is protected by copyright. All rights reserved. 
A

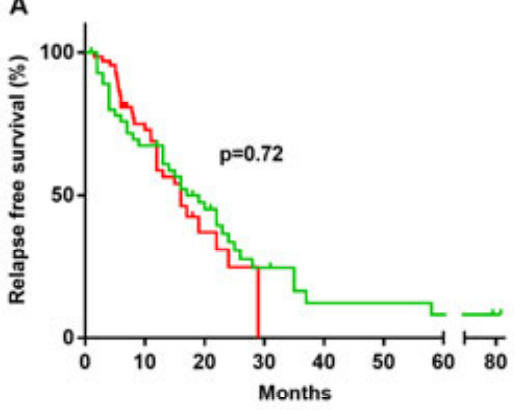

$\begin{array}{lllllllll}\text { — post RMT } & 57 & 33 & 19 & 8 & 4 & 4 & 3 & 1\end{array}$

- post single RTX $\begin{array}{cccccccc}67 & 38 & 8 & 0 & 0 & 0 & 0 & 0\end{array}$

A

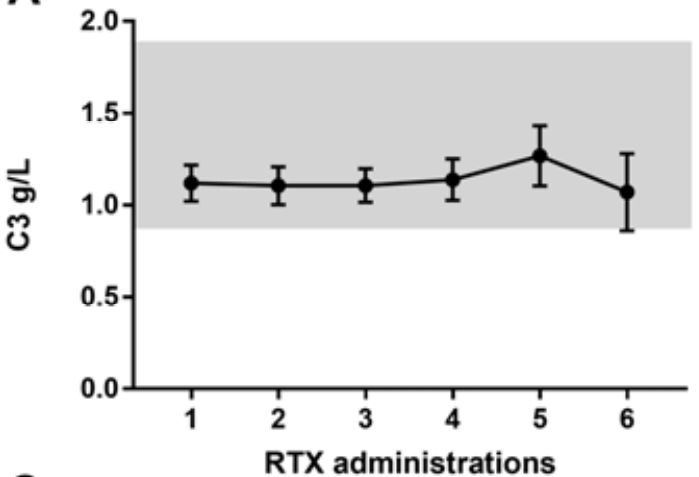

C



B

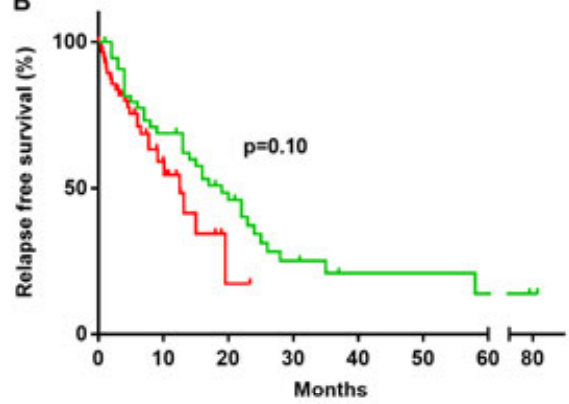

$\begin{array}{cllllllll}\text { - post RMT } & 57 & 33 & 19 & 9 & 4 & 4 & 3 & 2\end{array}$

B

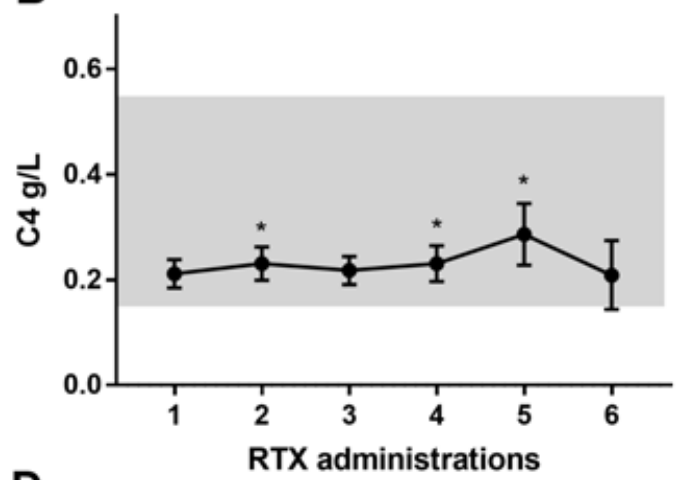

D

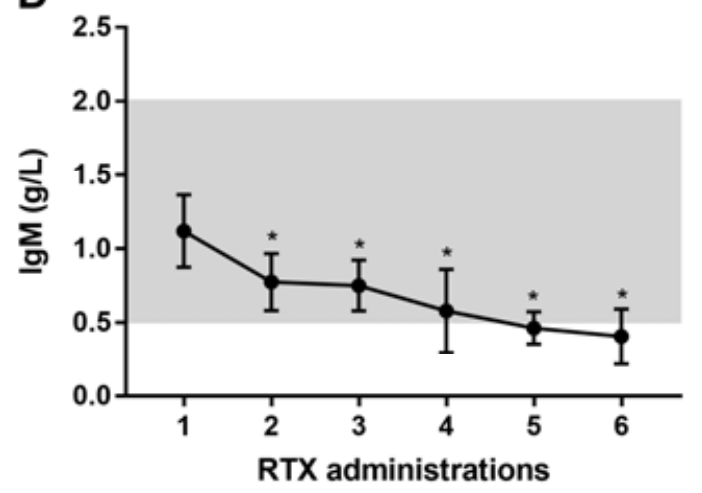

This article is protected by copyright. All rights reserved. 